\title{
SOME LIMIT THEOREMS FOR RATIOS OF TRANSITION PROBABILITIES
}

\section{RITA CHATTOPADHYAY}

Depariment of Mathematics

Eastern Michigan University

Ypsilanti, MI 48197

( Received October 8,1986 )

\begin{abstract}
In this paper, infinite dimensional forward convergent stochastic chains have been considered in the framework of [1]. The main result of this paper deals with the observation that the total flow of probability from the C-states to the Tstates is very small compared to that from the T-states to the C-states, if the chain is observed for a sufficiently long time. Some examples have been given to justify the assumptions involved.
\end{abstract}

KEY WORDS AND PHRASES. Infinite stochastic matrices, convergence, basis, C-states, $\mathrm{T}$-States.

1980 AMS SUBJECT CLASSIFICATION CODE. $60 \mathrm{~J}$.

\section{IRTRODUCTION.}

Let $\left(P_{n}\right)$ be a sequence of finite or countably infinite stochastic matrices. Then $\left(P_{n}\right)$ is called a convergent chain iff for each $k \geq 1, \lim _{n \rightarrow \infty} P_{k, n}=Q_{k}$ exists and is a stochastic matrix. Here $P_{k, n}=P_{k+1} P_{k+2} \ldots P_{n}$ for $k<n$. It has been proved in [1] that for such a chain there exists a unique partition $\left\{\mathrm{T}, \mathrm{C}_{1}, \mathrm{C}_{2}, \ldots\right\}$ of the state space $S$ such that for any limit point $Q$ of the $Q_{k}^{\prime}$ s the following are true:

$$
\begin{aligned}
Q_{i j} & =0, \text { for all } i \text { if } j \varepsilon t \\
& =0 \text {, if } i \text { and } j \text { belong to different C-classes } \\
& =Q_{k j}, \text { if } i, j, k \text { belong to the same C-classes. }
\end{aligned}
$$

One of the problems, here, is to identify the T-states and the C-states for infinite convergent chains. Also, the following theorem is known (see [2]) for finite chains.

THEOREM 1.1. Let $\left(P_{n}\right)$ be a finite convergent chain with basis $\left\{T, C_{1}, C_{2}, \ldots, C_{p}\right\}$. Let $L \mathrm{~T}$. Then for each positive integer $k \geq 1$,

$$
\lim _{m \rightarrow \infty} \frac{\sum_{n=k}^{m}\left(P_{n}\right)_{T} C_{L}}{\sum_{n=k}^{m}\left(P_{n}\right) L L^{c}}=0
$$

where $\left(p_{n}\right)_{A B}=\sum_{\substack{i \varepsilon A \\ j \in B}}\left(p_{n}\right)_{i j} \cdot$ 
The above theorem and some other results have been extended to the infinitedimensional case with some modifications. Some examples have been discussed to justify the assumptions involved.

2. PREL IMINARIES.

Condirion (U). A convergent, forward stochastic chain $\left(P_{n}\right)$ is said to satisy condition (U) if for each $j$ in each C-class in the basis,

$$
\lim _{k \rightarrow \infty} \operatorname{Sup}_{i \in T}\left|\left(Q_{k}\right)_{i j}-Q_{i j}\right|=0
$$

This condition yielded a number of finite space like results for infinite chains in [1]. However, the next few examples demonstrate that Theorem 1.1 fails to go over directly to the infinite state space, even for infinite chains which satisfy condition (U).

EXAMPLS. (a) For a convergent, infinite chain with basis $\left\{\mathrm{T}, \mathrm{C}_{1}, \mathrm{C}_{2}, \ldots\right\}$ and any $L \quad T$ the ratio

$$
\frac{\sum_{q=k}^{m}\left(p_{q}\right)_{T} c_{L}}{\sum_{q=k}^{m}\left(P_{q}\right)_{L L} c}
$$

for $k \geq 1$, may not go to zero as $m \rightarrow \infty$. For, if

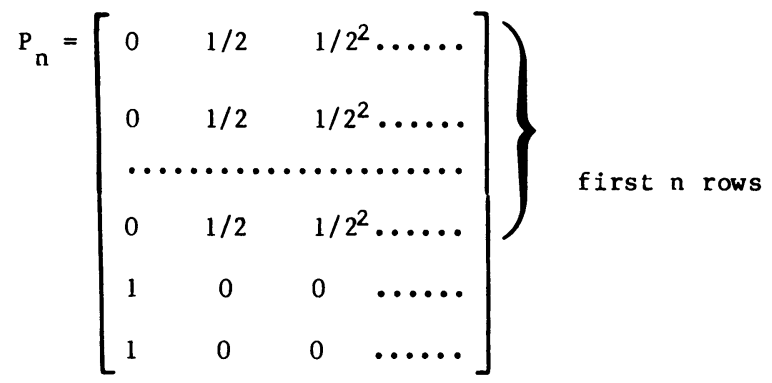

then it has been shown in [1], that for each $k$,

$$
\lim _{n \rightarrow \infty} P_{k, n}=\left[\begin{array}{ccc}
0 & 1 / 2 & 1 / 2^{2} \\
0 & 1 / 2 & 1 / 2^{2} \ldots \ldots \\
\ldots \ldots \ldots \ldots & \ldots \ldots \ldots \ldots \ldots
\end{array}\right]
$$

The basis of this chain is of course $\{T=\{1\}, C=\{2,3, \ldots\}\}$. But for all k<m,

$$
\frac{\sum_{n=k}^{m}\left(P_{n}\right)_{T} c,\{1\}}{\sum_{n=k}^{m}\left(P_{n}\right)\{1\}, T^{c}}=\infty
$$


It can be seen that this chain satifies a condition stronger than (U) because $\left\|Q_{k}-Q\right\|=0$ for all $k$.

(b) This example is given to show that the previous limit may be finite but not zero. If $a_{k}=2^{4(k-1)}, k \geq 1$ with $a_{k} \leq n<a_{k+1}$, let

$$
\begin{aligned}
& \left(P_{3 n-2}\right)_{i j}= \begin{cases}1 / 2^{j} & \text { if } i=0, j \geq 1 \\
1-1 / 2^{k} & \text { if } 1 \leq i=j \leq k \\
1 & \text { if } i=j \geq k+1 \\
1 / 2^{k} & \text { if } j=n \text { and } 1 \leq i \leq k\end{cases} \\
& \left(P_{3 n-1}\right)_{i j}= \begin{cases}1 & \text { if } 0 \leq 1=j \leq k \\
1 & \text { if } j=n, i>k\end{cases} \\
& \left(P_{3 n}\right)_{i j}= \begin{cases}1 & \text { if } i=n, j=0 \\
1 & \text { if } i=j \neq n\end{cases}
\end{aligned}
$$

Here, the state space is $\{0,1,2, \ldots\}$. It has been shown in [1] that

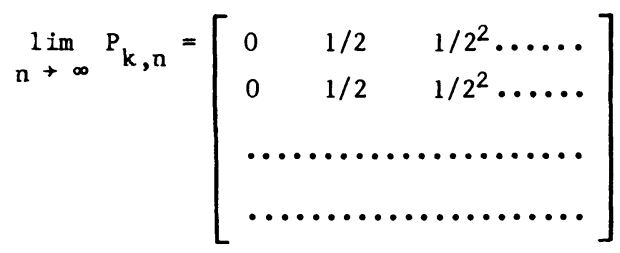

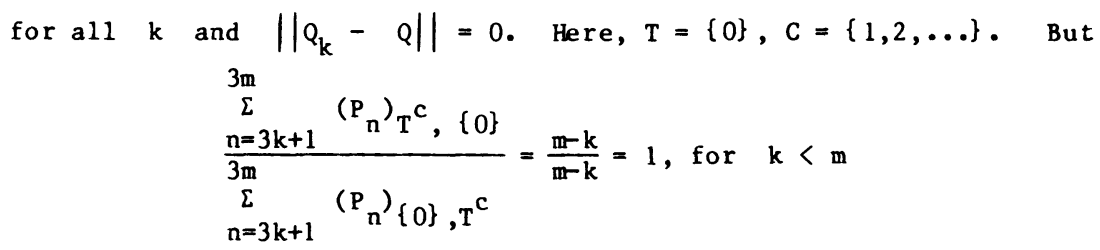

so that

$$
\lim _{m \rightarrow \infty} \frac{\sum_{n=k}^{m}\left(P_{n}\right) T^{c},\{0\}}{\sum_{n=k}^{m}\left(P_{n}\right)_{\{0\}, T}}=1 \text {, for } a 11 k
$$

so that the limiting value of the above ratio is not zero.

\section{MAIN RBSULTS.}

LEMMA 3.1. Let $\left(P_{n}\right)$ be any chain (finite or infinite). Also, let 
$T=\left\{j \mid \lim _{\rightarrow \infty}\left(p_{m, n}\right)_{i j}=0\right.$, for all $m$ and for all i $\}$

and assume that $Q_{k}=\underset{1+\infty}{\operatorname{fim}_{1+\infty}} P_{k, 1}$ are stochastic matrices. Then we have

$$
\sum_{n}\left(P_{n}\right)_{L L} c=\infty \text {, where }\left(P_{n}\right)_{L L} c=\sum_{\substack{i \varepsilon L \\ j \in L}}\left(P_{n}\right)_{i j} \text { and } L \quad T \text {. }
$$

PROOF. Suppose that the lemma is false. Then there exists $L$ $T$ such that $\sum\left(P_{n}\right)_{L L} c<\infty$. Then for given $\varepsilon>0$, there exists $k(\varepsilon)$ such that for all $k \geq k(\varepsilon)$,

$$
\sum_{n} \geq k\left(P_{n}\right)_{L L} c<\varepsilon .
$$

For any $t \in L,\left(P_{k, n}\right)_{t L} c=\left(P_{k+1} P_{k+1, n}\right)_{t L} c$

$$
\begin{aligned}
& =\sum_{i \varepsilon S}\left(P_{k+1}\right)_{t i}\left(P_{k+1, n}\right)_{i L} c \\
& =\sum_{i \varepsilon L}\left(P_{k+1}\right)_{t i}\left(P_{k+1, n}\right)_{i L} c+\sum_{i \varepsilon L} c\left(P_{k+1}\right)_{t i}\left(P_{k+1, n}\right)_{i L} c \\
& \leq \max _{i \varepsilon L}\left(P_{k+1, n}\right)_{i L} c+\left(P_{k+1}\right)_{L L} c .
\end{aligned}
$$

Therefore, $\max _{t \in L}\left(P_{k, n}\right)_{t L} c \leq \max _{t \varepsilon L}\left(P_{k+1, n}\right)_{t L} c+\left(P_{k+1}\right)_{L L} c$.

Repeating the procedure,

$$
\max _{t \varepsilon L}\left(P_{k, n}\right)_{t L} c \leq \sum_{j=1}^{n}\left(P_{k+j}\right)_{L L} c
$$

Therefore, for $k \geq k(\varepsilon)$ and $t \varepsilon L$,

$$
\sum_{j \in L} c\left(Q_{k}\right)_{t j}<\varepsilon
$$

which is a contradiction. Hence the supposition is false and the result follows.

REMAk 3.2. If $\pi_{k_{0}}$ is an infinite-dimensional initial probability row-vector and $\left(P_{n}\right)$ a sequence of infinite stochastic matrices, then let $\pi_{m}=\pi_{k_{0}} P_{k_{0}}, m \cdot$ If ${ }^{\pi} k_{0}$ has all positive entries, then for each $j \varepsilon T$, choosing $k_{0}$ sufficiently large it follows that for a convergent chain, $\lim _{n \rightarrow \infty} \pi_{n}(j)>0$.

Also, for $\mathrm{m}<\mathrm{n}$ and $i \varepsilon S$,

$$
\begin{aligned}
& \pi_{n+1}(i)-\pi_{n}(i)=\sum_{j \neq i} \pi_{n}(j)\left(P_{n+1}\right)_{j i}-\pi_{n}(i)\left[1-\left(P_{n+1}\right)_{11}\right] \\
& \pi_{n}(i)-\pi_{m}(i)=\sum_{j \neq i} \sum_{k=m}^{n-1} \pi_{k}(j)\left(P_{k+1}\right)_{j i}-\sum_{k=m}^{n-1} \pi_{k}(i)\left[1-\left(P_{k+1}\right)_{1 i}\right]
\end{aligned}
$$


The above identities are use to get the following results:,

THEOREM 3.3. Let $\left(\mathrm{P}_{n}\right)$ be a convergent chain with basis $\left\{\mathrm{T}, \mathrm{C}_{1}, \mathrm{C}_{2} \ldots\right\}$.

(A) Suppose that tET. Then the following hold:

(i) $\sum_{n=1}^{\infty}\left[1-\left(P_{n}\right)_{t t}\right]=\infty$
(ii) For $j \in T, \frac{1 i m}{n+\infty} \frac{\left(P_{n}\right)_{j t}}{1-\left(P_{n}\right)_{t t}}=0$

(B) Suppose that there is a state tes such that

$$
\begin{aligned}
& \text { (i) } \sum_{n=1}^{\infty}\left[1-\left(P_{n}\right)_{t t}\right]=\infty \\
& \text { (ii) } \lim _{n+\infty} \frac{\left(P_{n}\right)_{j t}}{1-\left(P_{n}\right)_{t t}}=0 \text {, uniformly for } j \neq t \text {. }
\end{aligned}
$$

Then $t \varepsilon T$.

(C) Suppose that there is only one T-state such that

$$
\lim _{n \rightarrow \infty} \frac{\left(P_{n}\right)_{j t}}{1-\left(P_{n}\right)_{t t}}=A_{j}\left(0 \leq A_{j}<\infty\right) \text { for each } j \neq t \text {. }
$$

Then $t \varepsilon T$ iff $\left.\sum_{n=1}^{\infty}\left[1-P_{n}\right)_{t t}\right]=\infty$ and $A_{j}=0$ for each $j \neq t$.

PROOP. (A) Part (i) follows since $\left(P_{k, n}\right)_{t t} \geq\left(P_{k+1}\right)_{t t}\left(P_{k+2}\right)_{t t} \ldots \ldots \ldots\left(P_{n}\right)_{t t}$

$$
\text { So that } 0=\left(Q_{k}\right)_{t t} \geq \prod_{m=k+1}^{\infty}\left(P_{k+m}\right)_{t t}
$$

which means that $\sum_{n=1}^{\infty}\left[1-\left(P_{n}\right)_{t t}\right]=\infty$.

For part (ii), suppose that it is false for some $j_{0} \varepsilon T$. Then there exists $B>0$ and a positive integer $N$ such that for all $n \geq N$,

$$
\left(P_{n}\right)_{j_{0}} \geq B\left[1-\left(P_{n}\right)_{t t}\right]
$$

Then from equation (3.2) for $n>m>N$, we have

$$
\pi_{n}(t)-\pi_{m}(t) \geq \sum_{k=m}^{n=1}\left[\beta \pi_{k}\left(j_{0}\right)-\pi_{k}(t)\right]\left[\left(1-P_{k+1}\right)_{t t}\right]
$$


which is impossible because of part (i) and because the left side goes to zero as $\mathrm{m}, \mathrm{n}+\infty$ but $\lim _{\mathrm{im}} \pi_{k}\left(j_{0}\right)>0$ and $\lim _{\mathrm{k} \rightarrow \infty} \pi_{k}(t)=0$. Therefore the supposition is false and part (ii) $\stackrel{k \rightarrow \infty}{1 s}$ proved

(B) Suppose teT. Then there exists $n_{0}$ such that $n \geq n_{0}$ implies

$$
\pi_{n}(t)>2 B>0 \text {. }
$$

By (1),

$$
\begin{aligned}
& 2 \text { B }\left[1-\left(P_{n+1}\right)_{t t}\right] \\
& \leq \sum_{j \neq t} \pi_{n}(j)\left(P_{n+1}\right)_{j t}+\pi_{n}(t)-\pi_{n+1}(t) \\
& \leq \max _{j \neq t}\left(P_{n+1}\right)_{j t}+\pi_{n}(t)-\pi_{n+1}(t) .
\end{aligned}
$$

By condition ( $i i)$, there exists $N>n_{0}$ such that $n \geq N$ implies

$$
\max _{j \neq t}\left(P_{n+1}\right)_{j t}<B\left[1-\left(P_{n}\right)_{t t}\right]
$$

then we have from above, for $n>N$,

$$
\text { B }\left[1-\left(P_{n+1}\right)_{t t}\right] \leq \pi_{n}(t)-\pi_{n+1}(t)
$$

which means that $B \sum_{\mathrm{p}=\mathrm{N}+1}^{\mathrm{m}}\left[1-\left(\mathrm{P}_{\mathrm{n}+1}\right)_{t t}\right] \leq \pi_{\mathrm{N}+1}(t)-\pi_{\mathrm{m}+1}(t)<2$ which contradicts (i).

(C) This part follows immediately from parts (A) and (B).

THEOREM 3.4. Let $\left(P_{n}\right)$ be a convergent infinite chain with $\left\{T, C_{1}, C_{2}, \ldots\right\}$ as its basis. Then for all $k \geq 1$,

$$
\lim _{m \rightarrow \infty} \frac{\sum_{n=k}^{m}\left(P_{n}\right)_{B A}}{\sum_{n=k}^{m}\left(P_{n}\right)_{A A} c}=0
$$

where A (finite set) T,B (finite set) $T^{c}$. Moreover, if

$$
\left.\underset{\pi \rightarrow \infty}{\lim } \underset{t \in T}{\operatorname{linf}} \sum_{i \notin C_{s}}\left(Q_{n}\right)_{t i}\right]>0
$$

then the above result holds for any $A \subset T$ and finite $B \subset C_{s}$.

PROOF. If $\pi_{n}(A)=\sum_{t \varepsilon A} \pi_{n}(t)$ and $m<n$, then

$$
\left.\left.\pi_{n}(A)-\pi_{m}(A)=\sum_{t \in A} \sum_{j \neq t} \sum_{q=m}^{n-1} \pi_{q}(j)\right) P_{q+1}\right)_{j t}
$$




$$
\begin{gathered}
-\sum_{t \in A} \sum_{q=m}^{n-1} \pi_{q}(j) \sum_{j \neq t}\left(P_{q+1}\right)_{t j} \\
\geq \sum_{q=m}^{n-1} \sum_{j \in B} \pi_{q}(j)\left(P_{q+1}\right)_{j A}-\sum_{q=m}^{n-1} \sum_{t \in A} \pi_{q}(t)\left(P_{q+1}\right)_{t A} c
\end{gathered}
$$

Now, for all $t \varepsilon A$ (finite) and $j \in B$ (finite) and $q$ sufficiently large,

$$
\pi_{q}(t)\left\langle\varepsilon \varepsilon_{1} \text { and } \pi_{q}(j)>\varepsilon_{1}\right. \text {. }
$$

Therefore, for $m$ sufficiently large and $m<n$,

$$
\pi_{n}(A)-\pi_{m}(A)>\varepsilon_{1} \sum_{q=m}^{n-1}\left(P_{q+1}\right)_{B A}-\varepsilon \varepsilon_{1} \sum_{q=m}^{n-1}\left(P_{q+1}\right)_{A A} c
$$

Let $\left(m_{r}\right)$ by any given sequence of positive integers. Then there exists $\left(p_{r}\right)\left(m_{r}\right)$ such that for each $r \geq k$, there exists $B>0$ such that

$$
\begin{array}{ll}
\sum_{\mathrm{q}=\mathrm{k}}^{\mathrm{r}}\left(\mathrm{P}_{\mathrm{q}}\right)_{\mathrm{BA}} \leq \varepsilon / 2 \sum_{\mathrm{q}=\mathrm{k}}^{\sum_{\mathrm{r}}}\left(\mathrm{P}_{\mathrm{q}}\right)_{\mathrm{AA}} \mathrm{c} \\
\text { since } & \sum_{\mathrm{q}}\left(\mathrm{P}_{\mathrm{q}}\right)_{\mathrm{AA}} \mathrm{c}=\infty \text { and } \sum_{\mathrm{q}=\mathrm{r}}^{\mathrm{P}_{\mathrm{r}}}\left(\mathrm{P}_{\mathrm{q}}\right)_{\mathrm{AA}} \mathrm{c}>B>0 .
\end{array}
$$

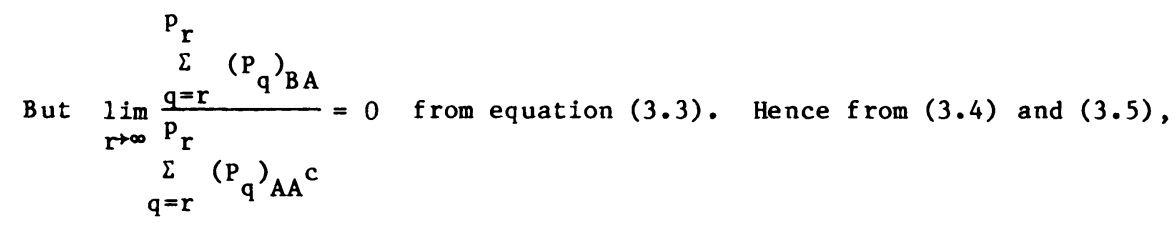

for sufficiently large $r$, we have

$$
\sum_{q=k}^{p_{r}}\left(P_{q}\right)_{B A}<\varepsilon \sum_{q=k}^{p_{r}}\left(P_{q A A}\right)_{A A}
$$

and the first part of the theorem follows.

We refer the reader to a result obtained in [2] for the proof of the second part. It has been proved in [2] that for all $i \in C_{s}$,

$$
\sum_{n=1}^{\infty} \sum_{t \in A}\left(P_{n}\right)_{i t}<\infty
$$

Using the above result, the proof of the second part easily follows.

EXAMPL 3.5. We now give an example to show that in Theorem 3.4 , the condition that the flow in the denominator is from $A$ to $A^{c}$, is necessary. 
380

R. CHATTOPADHYAY

$$
\text { Let } \mathrm{P}_{\mathrm{n}}=\left[\begin{array}{ccccc}
0 & 1 / \mathrm{n} & 1-1 / \mathrm{n} & 0 & \ldots \ldots \ldots \\
1 / \mathrm{n} & 1-1 / \mathrm{n} & 0 & 0 & \ldots \ldots \\
0 & 1 & 0 & 0 & \ldots \ldots \\
0 & 1 & 0 & 0 & \ldots \ldots
\end{array}\right]
$$

By Bernstein's theorem, the products are weakly ergodic. In fact,

$$
\begin{aligned}
\left(P_{k, n+1}\right)_{i 1} & =\sum_{s}\left(P_{k, n}\right)_{i s}\left(P_{n+1}\right)_{s 1} \\
& =\frac{1}{n+1}\left(P_{k, n}\right)_{i 2}+0 \text { as } n \rightarrow \infty \text {, for a1- } \\
\left(P_{k, n+1}\right)_{i 2} & =\sum_{\left(P_{, n}\right)_{i s}\left(P_{n+1}\right)_{s 2}} \\
& =1-\left(P_{k, n}\right)_{i 1}+\frac{1}{n+1}\left(P_{k, n}\right)_{i 1}-\frac{1}{n+1}\left(P_{k \cdot n}\right)_{i 2} \\
& +1 \quad \text { as } n \rightarrow \infty .
\end{aligned}
$$

So, the chain is strongly ergodic with $T^{c}=\{2\}$. But if $A=\{1\}, B=\{2\}$

$$
\text { then } \frac{\sum_{n=k}^{m}\left(P_{n}\right) 21}{\sum_{n=k}^{m}\left(P_{n}\right)_{12}}=1 \text {, for all } k \leq m \text {. }
$$

Hence, the assertion is true.

\section{REFERENCES}

1. MUKHERJEA, A. and NAKASSIS, A., Convergence of Non-homogeneous Stochastic Chains With Countable States, J. Multivariate Analysis Vo1. 16, No. 1 (1985), 85-117.

2. MUKHERJEA, A., NAKASSIS, A. and ISSAACSON, D., Determination of the Basis of a Non-homogeneous Ma rkov Chain, Statistics and Decision 2 (1984), 363-375.

3. MUKHERJEA, A., A New Result on the Convergence of Non-homogeneous Stochastic Chains, Transactions of the A.M.S. Vo1. 262, No. 22 (1980), 505-520.

4. ISAACSON, D.L. and MADSEN, R.W., Markov Chains: Theory and Applications, J. Wiley, New York. 


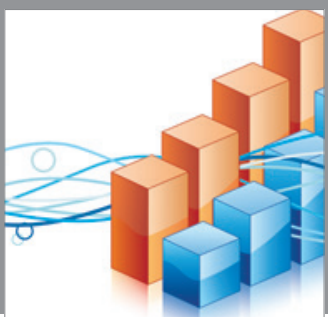

Advances in

Operations Research

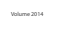

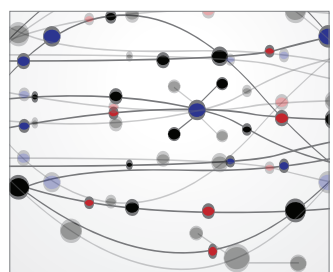

\section{The Scientific} World Journal
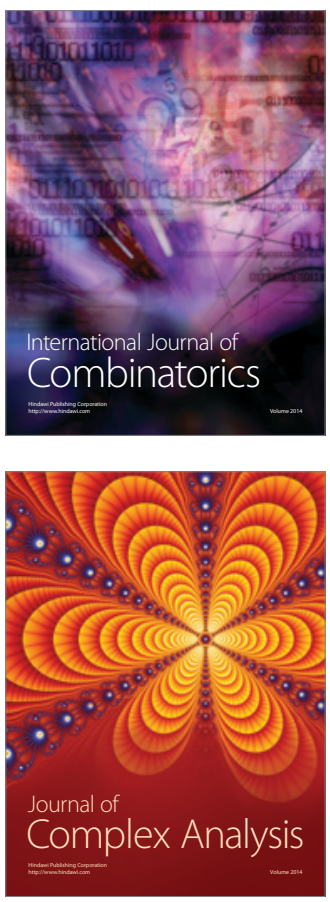

International Journal of

Mathematics and

Mathematical

Sciences
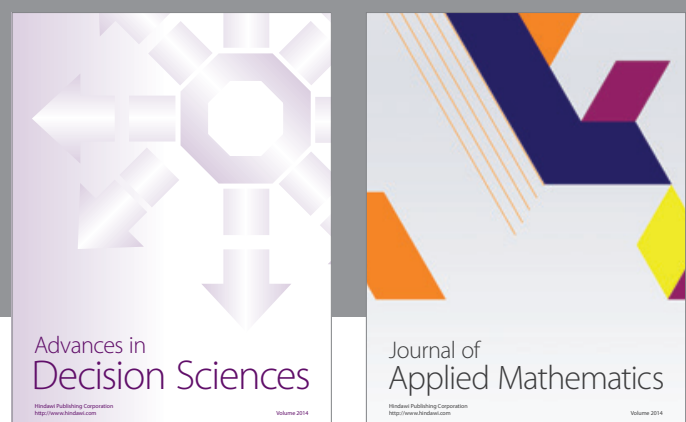

Journal of

Applied Mathematics
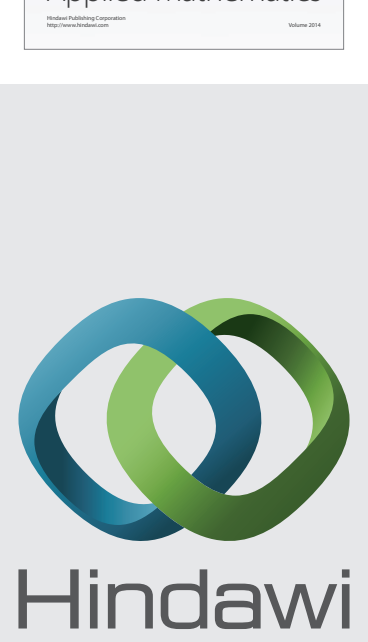

Submit your manuscripts at http://www.hindawi.com
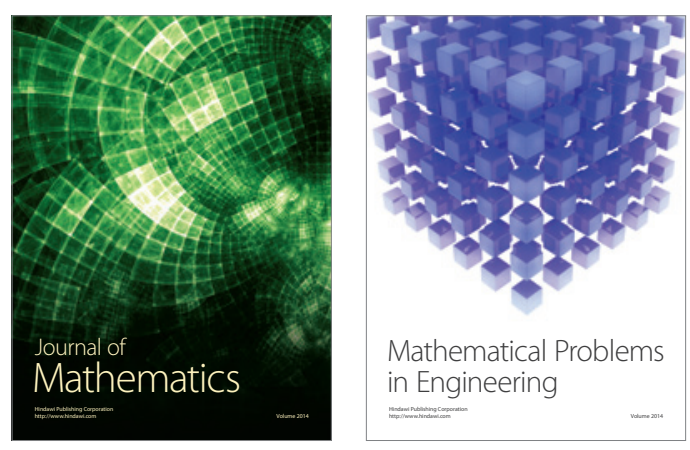

Mathematical Problems in Engineering
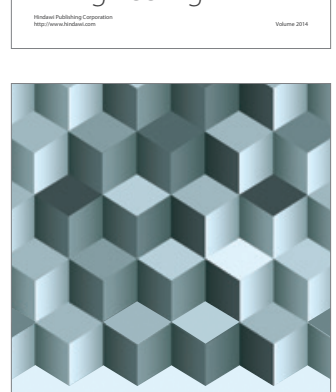

Journal of

Function Spaces
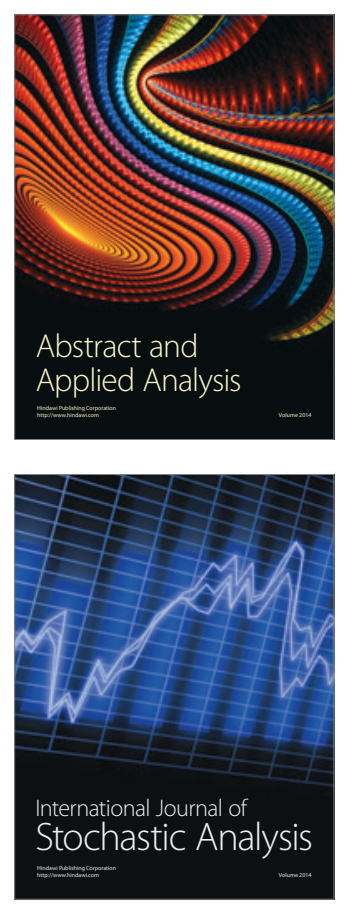

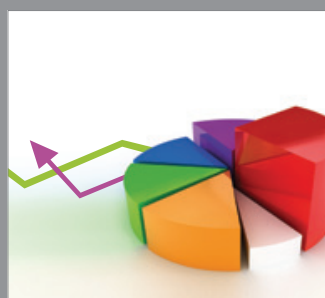

ournal of

Probability and Statistics

Promensencen
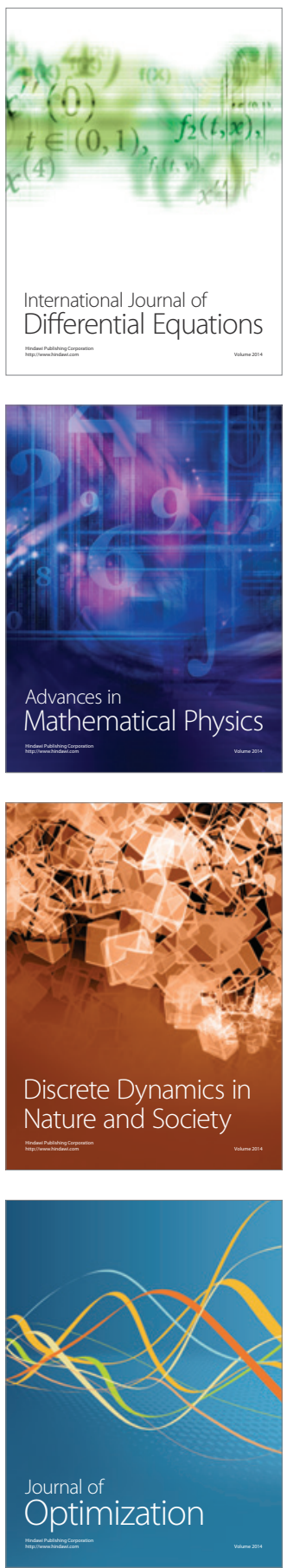